\title{
ON THE RELICT SCALES AND MELODIC STRUCTURES IN THE SETO SHEPHERD TUNE KAR'AH $\ddot{A} \ddot{A} L$
}

\author{
Žanna Pärtlas
}

\begin{abstract}
Our knowledge of the past is inevitably fragmentary, especially if we speak about such an 'immaterial' subject as music. The only possibility to unveil the sounds of the past is to 'excavate' information from contemporary performances, archival sound recordings, and musical transcriptions. Some older musical genres that preserved their ancient features until the era of sound recording provide the best opportunities for the research of the traditional musical thinking, including its deepest roots. The Seto shepherd songs, which are the subject of the analysis in this article, constitute one of such genres.

This research concentrates on two aspects of the musical structure of the Seto shepherd tune kar'ahääl: the structure of musical scales and the melodic contours. The material of analysis is the collection by Anu Vissel, Eesti karjaselaulud I. Setu karjaselaulud (Estonian Shepherd Songs I. Setu Shepherd Songs, 1982), which consists of transcriptions of 99 shepherd songs; about 90 of them are variants of the same tune type. According to this publication, the intervallic structures of the shepherd tune's scales are extremely various; there are also many different melodic contours. The present research aims to reveal the system behind the various scale structures and melodic contours, and, on this ground, tries to reconstruct the possible processes of formation of the musical scales and melody in this particular tune and make some hypotheses about such processes in early traditional music.
\end{abstract}

Keywords: early traditional music, melodic contours, musical analysis of traditional music, musical scales, musical thinking, Seto song tradition, shepherd songs

Contemporary ethnomusicology, ${ }^{1}$ which inherited many of its ideas from musical anthropology, is largely concerned with the present day. The present is both interesting and 'convenient' for ethnomusicological research: interesting because of its actuality and immediacy, and 'convenient' because researchers have every possibility to gather ideal material for their investigations - material that is both full and rich in context. However, though our knowledge of the past is inevitably fragmentary, especially if we speak about such an 'immaterial' subject as music, a fascination with the past is an inherent feature of human nature, and the past history of musical culture is no exception. 
What can we know about our musical past and where are the sources of such knowledge? Music archaeology can provide some evidence about the musical practices of the remote past on the basis of material sources such as excavated artefacts, early musical notations, and theoretical writings. Such investigations are not able, however, to discover what was actually played or sung or how the music actually sounded. The only possibility to unveil the sounds of the past is to 'excavate' information from contemporary performances and archival sound recordings and musical transcriptions, which, when appropriately analysed, can provide evidence regarding traditional musical thinking, including its deepest roots. Some older musical genres that preserved their ancient features until the era of sound recording provide the best opportunities for such research. Even today, indeed, musical genres still exist that are real 'portals to the past'. The Seto shepherd songs, which are the subject of the analysis in this article, constitute one of such genres.

The song tradition of the Seto (a small ethnic group of Estonians living in the south-east of Estonia and the adjoining territories of the Pskov region of Russia) provides very valuable source material for the investigation of the musical past. In the Seto song tunes one can find several structural features that point to their very old origin. Among such relicts of the ancient musical system, it is the Seto scales that are of the greatest interest. The most intriguing of these is the one-three-semitone mode (Sarv 1980; Pärtlas 1997, 2006a, 2010a) characteristic of the older genres of the Seto multipart songs, but the unusual scales also occur in the solo repertoire such as the shepherd songs. These scales and the pitch organisation of the Seto solo vocal genres in general was not, until now, the object of detailed ethnomusicological research. The purpose of this article is to uncover and to analyse the features of ancient musical thinking in the musical scales and melodic contours of the Seto solo shepherd songs.

It is no surprise that traces of ancient musical thinking manifest themselves especially distinctly in the Seto shepherd songs as this genre demonstrates an archaic musical style in many other traditional cultures. The main reason for this is evidently the strong connection of the shepherd songs to their extramusical functions, among which the communicative functions (signals, calls) are very important. In general, two types of communication can be found in the shepherd songs: communication with domestic animals, which is usually a phenomenon on the borders of music and onomatopoeia (the imitation of animal sounds), and long-distance communication with other shepherds (the herding calls). The shepherd songs also served as entertainment and consolation during the shepherds' long and lonely days. The abovementioned functions clearly influence the musical structure of the shepherd tunes and the manner of singing. The prolonged notes at the end of the melostrophes and the intensive 
singing voice both point to the original calling function of the Seto shepherd tune. However, the texts of the Seto shepherd songs often have a plaintive content and resemble laments - the shepherd complains about the hard and boring job, bad weather, and desolation. In the Seto shepherd songs there is also a musical resemblance with the lament (Vissel 1982: 4), another traditional genre that has preserved well the features of an ancient musical style.

The Seto shepherd songs have been well documented during the whole period of sound recording, from the beginning of the twentieth century up to the present. This research is based on the largest publication of Seto shepherd songs in notation, Eesti karjaselaulud I: Setu karjaselaulud (Vissel 1982). According to the statistics made by Anu Vissel herself, the collection consists of transcriptions of 99 shepherd songs performed by 80 different singers from 53 villages, which were recorded by 27 collectors over the period 1912-1979 (ibid.: 3). Some of these notations are aural transcriptions made by different collectors without the use of sound recording techniques, or where the sound recordings were lost or destroyed over time. In many cases the original sound recordings are preserved in various archives (mainly the Estonian Folklore Archives), and the musical transcriptions were made by Anu Vissel and overseen by Vaike Sarv (ibid.).

Although it would have been possible in many cases, I decided not to compare the transcriptions with the original sound recordings to check their reliability, using in my analysis the transcriptions in their published form. The partial control gave me the impression that the notations that were made on the basis of sound recordings generally reflect the actual sound of the songs. The fact that this collection consists of the notations made by several transcribers also allows to assume a sufficient degree of objectivity. However, having had sufficient aural experience with the real sound of the Seto shepherd songs, I am completely aware of the fact that it cannot be precisely conveyed by the Western musical notation, based on the 12TET (12-tone equal temperament) system. It is also obvious that musical perception of the transcribers may be influenced by their musical and educational background and that some 'human' mistakes and differences in interpretation are inevitable in the notation of traditional music.

Nevertheless, I believe that musical transcriptions made by our predecessors should not be neglected as a source of information and, being used with due reservation, can provide valuable material for investigation. In the case of my research, the advantage of the use of the written sources offered a possibility to involve in the analysis the large (almost comprehensive) amount of research material. I also proceeded from the conviction that an inevitable approximateness of the transcriptions does not seriously affect the results of the present analysis, as the performance of Seto shepherd songs itself is characterized by some approximateness of intonation, which means that the small pitch deviations 
have no significant emic value in this tradition. A critical analysis of the sources and acoustical measurements of the sound recordings would certainly be the object of a further investigation; however, in this essay, I would like to use the research potential of the existing written sources.

For my analysis I selected 82 performances, all of which are variants of the same tune type. Some transcriptions from Vissel's publication were rejected as they did not belong to the usual tune type (among the published transcriptions, for example, there are some solo performances of choral herding songs). I carried out a statistical analysis with regard to the use of the musical scales and compared the different scale structures to find logical relations between them. I also compared the melodic variants in order to reveal the underlying model of the tune type and the mechanisms of its realization in various performances (including the formation of the scales).

The analysis of the musical scales may appear, at first sight, to be rather old-fashioned as a formal procedure and way of describing the musical material. This is the reason why this theme, especially at the general theoretical level, was not much investigated in the recent ethnomusicological publications. ${ }^{2}$ However, the phenomenon of musical scale is one of the most important universals of human musical thinking, and interesting discoveries can certainly be made in this domain. The most problematic aspect of the conventional analysis of the traditional music scales appears to be the description of the scale structures using the names of the diatonic or chromatic scale notes and the measurement of the intervals between the scale notes in semitones. In fact, there are many musical traditions (especially the older ones) which are not based on the 12TET system, and the Seto ancient song tradition is among them. This problem should necessarily be recognized by the scholars dealing with this topic. All the same, we cannot describe and compare the scales if we do not have a unit of measurement. The vernacular music theories have usually no answer to this question; moreover, there are many musical systems where pitch relations are not proportional (i.e. have no unit in which all melodic and harmonic intervals could be measured). ${ }^{3}$ In this situation, some standard unit of measurement needs to be chosen, and the most convenient one seems to be a semitone. ${ }^{4}$ For more precise measurements we can also use the cents, but doing so we should not forget that a cent is nothing else than one-hundredth of a semitone; thus, measuring in cents, we still measure in semitones. In accordance with the aforesaid, I will use in my analysis the measurements in semitones, bearing in mind that in this case a semitone is nothing more than a conventional standard unit.

With respect to the musical peculiarities of the Seto shepherd songs, analysis of the scale structures seems to be a highly relevant aspect of research. Speaking more generally about the Seto song tradition, it should be noted that the Seto 
musical scales merit close analysis not only because of their unusual intervallic structures, but also because of the coexistence of the different scale types in the same song tradition, the formation of the mixed and transitional scale structures, and, especially, because of the use of different scales within the same tune types. Thus the Seto women can perform the same multipart song with the one-three-semitone, anhemitonic, anhemitonic-diatonic, and purely diatonic scales, not to mention transitional scale forms (for details see Pärtlas $2006 \mathrm{~b}, 2010 \mathrm{~b})$. This fact points to an early, dynamic musical system, in which the stability of the pitch organization is not yet achieved.

In the solo shepherd songs this tendency manifests itself even more strongly than in the multipart choral songs. Thus, according to my statistical analysis based on the publication by Anu Vissel, the same tune type was recorded with 15 different repeatedly used scales and a further 19 scales which were used only once; these were differentiated both by the number of scale notes and by the intervals between them. These figures are very large, even in the context of the Seto song tradition. It would be natural to suppose that such a big amount of scale structures found in the transcriptions may be partly explained by the imperfection of the Western notation and the differences in musical perception of the transcribers; ${ }^{5}$ however, these data are quite unusual, and it most probably points to both the unusually great variability of the Seto shepherd tune's scales and their significant distinction from the 12TET system. Such exceptional musical material provides the opportunity to examine the formation of the musical scales, a phenomenon which also proves illuminating in terms of the historical processes of musical development.

\section{ANALYSIS OF THE SCALES AND MELODIC CONTOURS}

Before presenting the results of the analysis, the tune type in question should be briefly described. All Seto solo shepherd songs use the same tune type, which is called by the bearers of the tradition kar'ahääl. The formal and rhythmic model of this tune type is stable enough for it to be easily recognized. Figure 1 provides a typical example. As can be seen, the melostrophe is composed of two lines, the melody of the second line generally repeating that of the first line. The text is not repeated. One very characteristic feature of the tune is the reduction of the last syllable of the second verse and the prolongation of the previous syllablenote (see the last note of the tune). The use of such long notes, which are sung in a very intensive manner and often with a specific change in vowel colour, creates the effect of a call (the final note in the melostrophes of the Seto songs is usually short). The number of syllables and notes in the lines can vary, but the rhythmic model presented in Figure 1 is the most typical one (Vissel 1982: 6). 


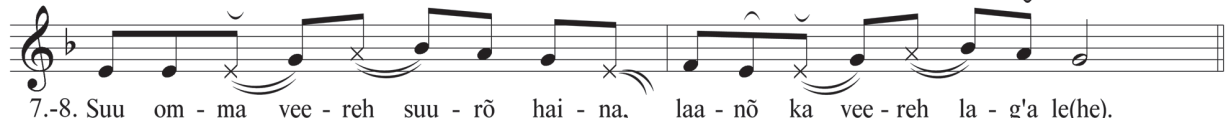

Figure 1. A shepherd song performed by Nati Tarkus (b. 1922) from Mikitamäe village in $1998 .^{6}$

\begin{tabular}{|c|c|c|c|}
\hline $\begin{array}{c}\text { Intervals in } \\
\text { semitones }\end{array}$ & $\begin{array}{c}\text { The way of } \\
\text { notation }(\boldsymbol{i n} \mathbf{G})\end{array}$ & The range & $\begin{array}{c}\text { The number of } \\
\text { performances }\end{array}$ \\
\hline $4-3$ & Eb-G-Bb & P5 & 7 \\
\hline $3-2$ & E-G-A & P4 & 6 \\
\hline $3-3$ & E-G-Bb & d5 & 2 \\
\hline $4-1-2$ & Eb-G-Ab-Bb & P5 & 8 \\
\hline $1-2-2$ & E-F-G-A & P4 & 8 \\
\hline $3-2-1$ & E-G-A-Bb & d5 & 8 \\
\hline $4-1-3$ & D-F\#-G-Bb & m6 & 5 \\
\hline $3-2-2$ & E-G-A-B & P5 & 4 \\
\hline $3-1-3$ & Eb-F\#-G-Bb & P5 & 3 \\
\hline $2-2-1$ & F-G-A-Bb & P4 & 4 \\
\hline $1-2-2-1$ & E-F-G-A-Bb & d5 & 3 \\
\hline $1-2-2-2$ & E-F-G-A-B & P5 & 3 \\
\hline $2-1-2-2$ & E-F $\sharp-G-A-B$ & P5 & 3 \\
\hline $2-2-1-2$ & Eb-F-G-Ab-Bb & P5 & 3 \\
\hline $2-2-2-1$ & Eb-F-G-A-Bb & P5 & \\
\hline & & & 3 \\
\hline
\end{tabular}

Figure 2. Scales occurring repeatedly in the notations from the collection of shepherd songs by Anu Vissel (1982). 
The results of the statistics concerning the use of the different scale structures are presented in the table in Figure $2 .{ }^{7}$ In the songs from Vissel's collection, 34 different scales were found. The table contains only the 15 scales that were used repeatedly. The 19 scales used only once each are not included in the table; they are, however, taken into account in the analysis. From the table one can see that three of the repeatedly used scales comprise three notes, seven scales four notes, and five scales five notes. No single scale prevails significantly over the others. However, the overall statistics reveal that most of the scales are 4-note scales, occurring in 46 performances out of 82. 3-note scales occur in 20 performances and 5 -note scales in 25 performances. The main tone of the scale (the 'principal tone'), which is also the final note of the tune, is always located in the middle of the scale (the note $G$ in the tables and musical examples), which is also a characteristic of Seto multipart songs. The similarity with the multipart song style also manifests itself in the use of the one-three-semitone scale: the scale 3-1-3 (Eb-F\#-G-Bb) is found three times and the related structure 4-1-3 (D-F\#-G-Bb) five times. The scales in the range of the diminished fifth are even more characteristic, occurring altogether in 13 performances. Among these, the structure 3-2-1 (E-G-A-Bb) is found eight times, while the similar structure 1-2-2-1 (E-F-G-A-Bb), where the 3-semitone interval E-G is filled by the scale note $\mathrm{F}$, occurs four times. The 3 -note scale in the range of the diminished fifth (which coincides with the diminished triad E-G-Bb) occurs only twice, but this is still notable and certainly not an accidental fact.

The table reveals, amongst other things, that many scales have intervals between adjacent scale notes that are wider than the major second (these are mostly minor and major thirds, more rarely the perfect fourth). In the discussion below, I will name such intervals 'wide intervals' or 'gaps'. The most notable fact is that the 3-note scales almost always have these wide intervals. Among them, the scales with two wide intervals are especially noteworthy: the structure 4-3 (Eb-G-Bb) occurs seven times, the structure 3-3 (E-G-Bb) twice, and the structure 5-3 (D-G-Bb) once. If there is only one wide interval in the 3-note scale, this is always located in the lower part of the scale, as in the structure 3-2 (E-G-A), which occurs six times, 4-2 (Eb-G-A) and 4-1 (Eb-G-Ab), each of which is found only once.

The majority of the 4-note scales also contain these wide intervals between adjacent scale notes (usually thirds). There are such structures as 4-1-2, 3-2-1, $4-1-3,3-2-2$, as well as others. Here we may note the same pattern as in the 3 -note scales: if there is only one wide interval in the scale, it is always in the lower part of the scale; if there are two wide intervals, the lower one is wider than the upper one or, more rarely, they are of the same size. It is possible that this rule is a manifestation of a more general feature of human musical 
thinking, an issue to which I shall return below. The 5-note scales are usually, so to speak, 'filled', i.e. they do not contain intervals between adjacent scale notes that are wider than a major second.

The column in the table (Figure 2) that shows the range of the musical scales reveals that the scales with different numbers of notes generally have the same range, most often a perfect fifth. This suggests that the scales with more notes may have developed over time from those with fewer notes by a process of filling the wide intervals in the latter with extra notes. Indeed, an analysis of the melodic contours and a comparison of the melodic variants give the impression that behind many of the variants based on the 4-note and 5-note scales there is a 3 -note proto-structure.

This last observation suggests that the most ancient versions of the Seto shepherd tune are those based on the 3-note scales with one or two wide intervals between the scale notes. It would appear that the juxtaposition of three pitch levels - lower, central (middle), and upper - reinforced by the largerthan-usual pitch contrast (in the cases where there are wide intervals between adjacent scale notes) is more important for the Seto traditional singers than the exact intervals between the scale notes. The significant pitch variability and the mutability of the scale notes are illustrated in Figure 3. The upper and lower scale notes can shift in relation to the central scale note to form different intervallic structures. Some of these structures seem to be more stable, as they occur in the notations more frequently. This is the case in two structures: the 4-3 structure, which coincides with the major triad $\mathrm{Eb}-\mathrm{G}-\mathrm{Bb}$, and the 3-2 structure, which is the anhemitonic trichord E-G-A. However, it is possible that the prevalence of these two structures in the published notations reflects more the transcribers' perceptions of the scales than the actual sound of the performances. The large number of micro-alteration symbols in the notations from Anu Vissel's collection is evidence of the great number of intermediate intervals between scale notes.

It is notable that the pitch of the scale notes varies not only in the performances of different singers, but also in different performances by the same singer and even during the same performance. Figure 4 presents three variants of the shepherd tune performed by the outstanding Seto singer Olga Laanetu (b. 1911) in 1971, 1973, and 1976 (Vissel 1982: 35-39). The first of these is transcribed with the scale $\mathrm{Eb}-\mathrm{G}-\mathrm{Bb}$ (the quasi 'major triad'), the second has the scale in the range of the diminished fifth $\mathrm{E}-\mathrm{G}-\mathrm{Bb}$ (the quasi 'diminished triad'), and the third has the scale E-G-A (i.e. the anhemitonic trichord). From the transcription of the earliest of the three performances we can also see that the pitch of the lower scale note was quite unstable (see the up arrows above the scale note $\mathrm{E} b$ ); listening to the sound recording of this performance, I have found that in the second half of the song the lower scale note changed eventually to E natural. 


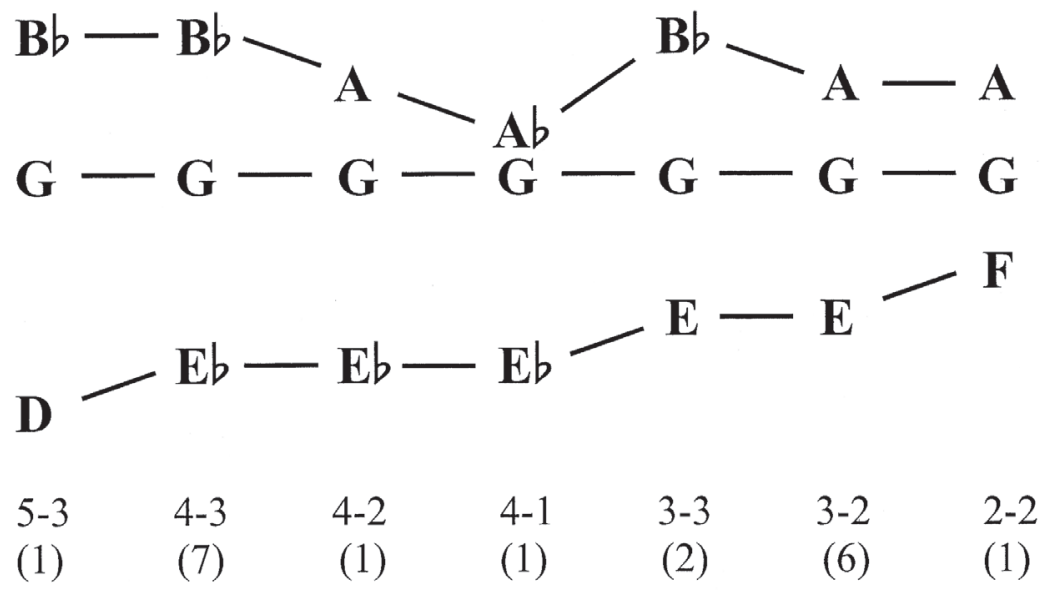

Figure 3. The mutability of the scale notes in the 3-note scales (the number of performances is given in brackets).

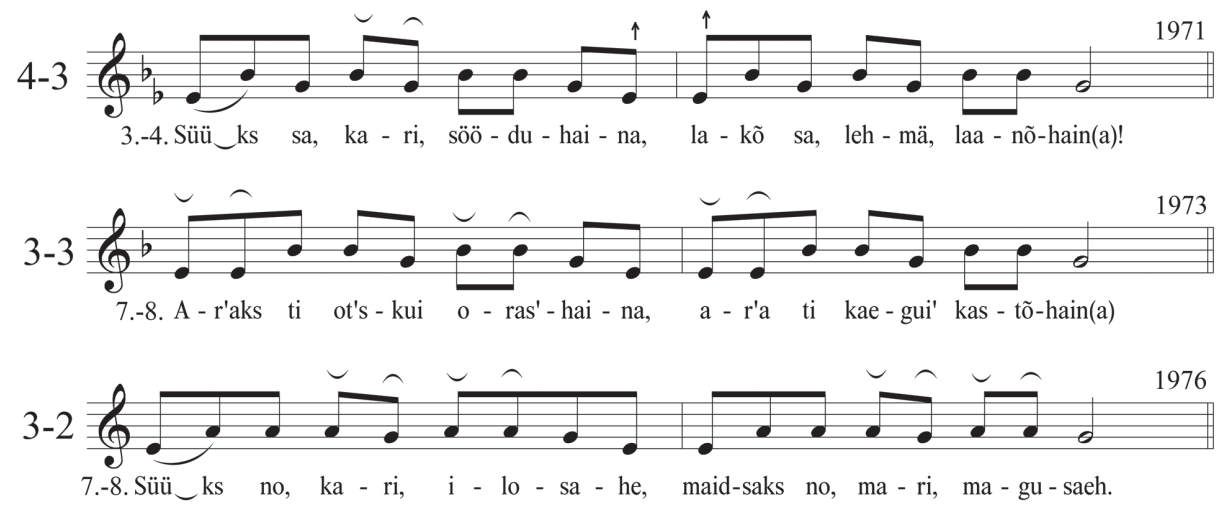

Figure 4. Three performances by Olga Laanetu (b. 1911), Suure-Rõsna village.

A similar variability in the scale structure may also be noted in the performances based on 4- and 5-note scales. The transcription of the shepherd tune sung by the most famous Seto singer, Anne Vabarna (1877-1964), in 1959 (Vissel 1982: 43-46) provides a good example of the variation of the scale notes during performance. In this notation the transcriber had to change the key signatures four times (the scale variants were as follows: D-E-G-Ab-B, D-E-G-Ab-Bb, D-Eb- 
$\mathrm{G}-\mathrm{A} b-\mathrm{B} b$, and $\mathrm{D}-\mathrm{E} b-\mathrm{G}-\mathrm{A} b-\mathrm{B})$, as well as use a large number of micro-alteration symbols (arrows) above the notes.

A similar variability is also found in the melodic contours of the Seto kar'ahääl. This suggests the need to search for the stable elements in the melodic structure, which allow us to recognize the tune type. In this paper, however, the analysis of the melodic contours deals not so much with the description of the tune type, but aims rather to reveal the mechanisms of the scale formation in the concrete realizations of the tune model. ${ }^{8}$

In searching for a method with which to analyse the melodic contours of the Seto shepherd songs, I decided to proceed from the 3-note (or 3-pitch level) model, which I assume to be the deep structure that underlies the majority of the particular variants of the tune irrespective of the number of notes in their scales. The comparison of the tune variants revealed some consistent patterns in the formation of the melodic contours, which will be demonstrated below.

In the present analysis, the melodic contour types are described by the use of three symbols: the letter $\mathrm{L}$ for the lower pitch level (the scale notes below the central tone), the letter $\mathrm{C}$ for the central tone (the 'principal tone' $\mathrm{G}$ ), and the letter $U$ for the upper pitch level (the notes above the central tone). Figure 5 provides three examples of the ascending-descending melodic contour LCUCL (the melodic fragments in the examples are the first lines of the melostrophes). Comparing the examples, one can notice that the points where the melody moves from one pitch level to another may be located in different positions with regard to the tune's rhythmic form. For example, in the first variant of the tune the melody moves from the lower level to the central at the fourth syllable-note, in the second variant this happens at the third syllable-note, and in the third variant at the second syllable-note; the melody reaches the upper level in these three variants respectively at the seventh, fifth, and sixth syllable-notes. The musical time for which the melody remains on each level also varies.

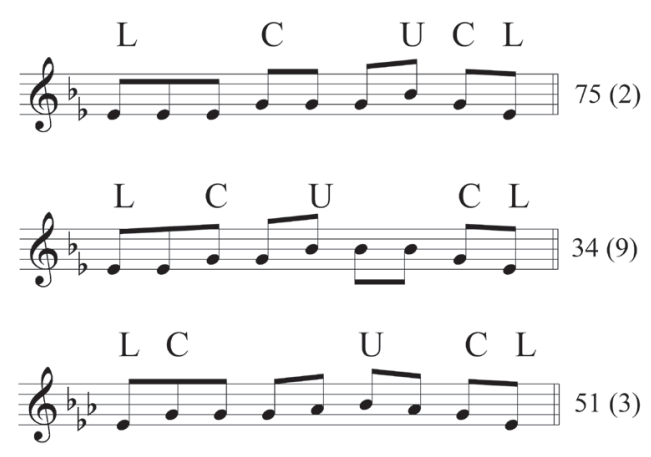

Figure 5. Three examples of the ascending-descending melodic contour LCUCL. ${ }^{9}$ 
Using these symbols, the melodic contours were described in all 82 variants of the shepherd tune under analysis. Statistics as to the use of each contour were then compiled, and these revealed a noticeable predominance of one melodic contour - the ascending-descending contour LCUCL which occurs 26 times (this melodic contour is shown in Figure 5). The table in Figure 6 comprises 12 melodic contours found in 70 performances; these are interpreted here as being systematic of the related structures. The melodic contours not included in the table occurred only once and would seem to be less characteristic. The order in which the melodic contours are placed in the table is meant to show the logic of their relationship to each other. Six contours begin at the lower pitch level (these clearly prevail, occurring in 48 performances), three contours begin at the central level (8 performances), and three contours at the upper level (13 performances). The 12 contours can be divided into four groups of three contours each (see the bold lines in the table); in each group the first contour is the most complex, with each subsequent contour differing from its predecessor by the omission of one level. The general logic of the melodic motion is to reach the upper pitch level (from beneath or directly) and then descend through the central level to the lower at the end of the phrase. Thus, all the melodic contours in the table end with the motion UCL, with the last two symbols CL usually corresponding to the last two syllable-notes of the phrase.

\begin{tabular}{|l|l|l|}
\hline & $\begin{array}{l}\text { The melodic contour of } \\
\text { the 1st line }\end{array}$ & $\begin{array}{l}\text { The number of } \\
\text { performances }\end{array}$ \\
\hline \multirow{2}{*}{ L } & LCUCLUCL & 1 \\
\cline { 2 - 3 } & LCUCUCL & 5 \\
\cline { 2 - 3 } & LCUCL & 26 \\
\cline { 2 - 3 } & LUCLUCL & 3 \\
\cline { 2 - 3 } & LUCUCL & 6 \\
\cline { 2 - 3 } & LUCL & 7 \\
\hline \multirow{2}{*}{ C } & CUCLUCL & 1 \\
\cline { 2 - 3 } & CUCUCL & 2 \\
\cline { 2 - 3 } & CUCL & 6 \\
\hline U & UCLUCL & 7 \\
\cline { 2 - 3 } & UCUCL & 3 \\
\cline { 2 - 3 } & UCL & 3 \\
\hline & & $\mathbf{7 0}$ \\
\hline
\end{tabular}

Figure 6. The melodic contours and their relations. 
We may also describe the relationship between the different melodic contours as a process of the gradual simplification of the contours from the most complex to the simplest model. This process is shown in Figure 7, where the model LCUCLUCL is gradually reduced to the model UCL. At every stage of simplification, the omitted (and, consequently, optional) pitch levels are designated by small letters in the scheme and by filled circles in the graphical depiction; the musical examples illustrate the possible melodic realizations of the contours in question. As the notations in Figure 7 show, the most elementary version of the Seto shepherd tune is simply the repetition of the upper scale note which leads to the descending end of the phrase. These musical examples also show how the melodic contours can be realized in the different scales.
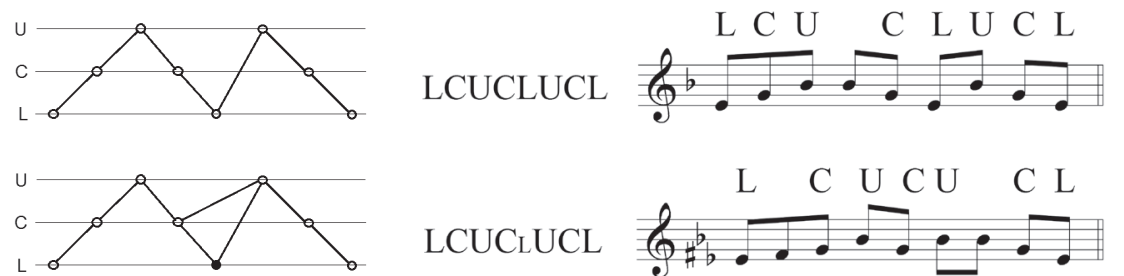

LCUCLUCL
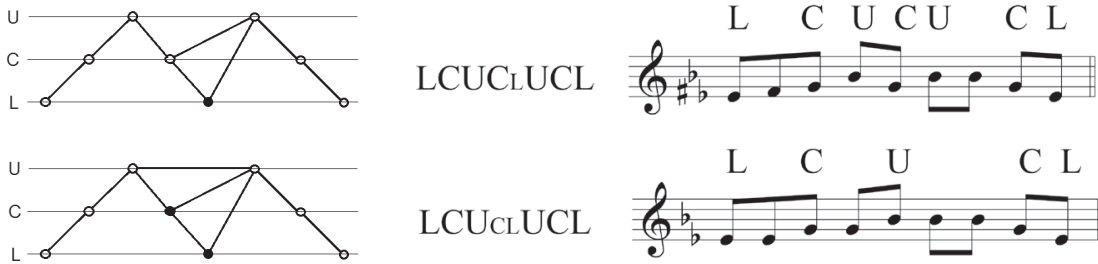

LCUCLUCL

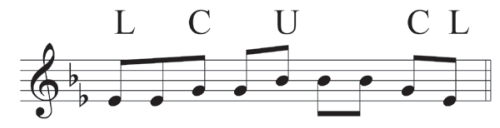
$34(9)$

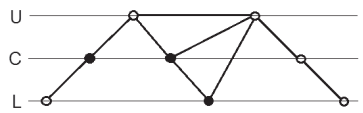

LCUCLUCL

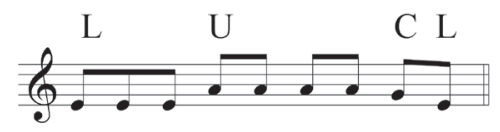
$22(1)$

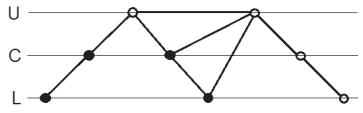

LCUCLUCL

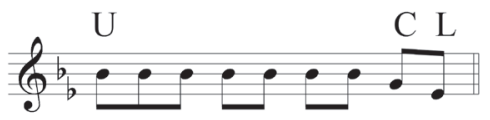

Figure 7. The process of the gradual simplification of the melodic contour.

The realization of the melodic models using scales with the different intervallic structures can be most clearly demonstrated by the examples with the same melodic contour. The use of the different scale notes in a similar melodic context is evidence of their interchangeability in the functional system of the mode. Sometimes we cannot even say whether they are actually different scale notes or merely different pitch variants of the same scale note. Some musical examples of this phenomenon are provided in Figures 8a, 8b, and 8c. Figure 8a, 
which comprises two variants of the melodic contour LCUCL, demonstrates the interchangeability of the scale notes $\mathrm{Bb}$ and $\mathrm{Ab}$ (the interchangeable notes are indicated by circles); in Figure 8b, which comprises two more variants of the same contour, the key signatures point to the interchangeability of the scale notes $\mathrm{E} / \mathrm{E} b$ and $\mathrm{A} / \mathrm{Ab}$. The three examples in Figure $8 \mathrm{c}$ are variants of the melodic contour LUCLUCL. Comparing these variants, we can find the notes $\mathrm{E} b / \mathrm{E} / \mathrm{F} \#, \mathrm{~A} / \mathrm{B} b / \mathrm{B}$ and $\mathrm{D} / \mathrm{E} b / \mathrm{E}$ in similar melodic contexts.

a) $\mathrm{Bb}=\mathrm{Ab}$
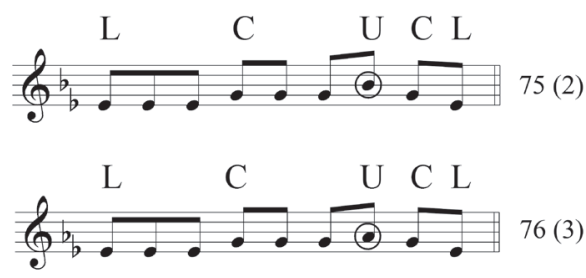

b) $\mathrm{E}=\mathrm{E} b, \mathrm{~A}=\mathrm{A} b$
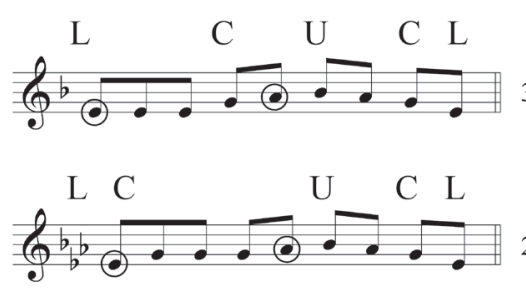

c) $\mathrm{E} b=\mathrm{E}=\mathrm{F} \#, \mathrm{~A}=\mathrm{B} b=\mathrm{B}, \mathrm{D}=\mathrm{E} b=\mathrm{E}$
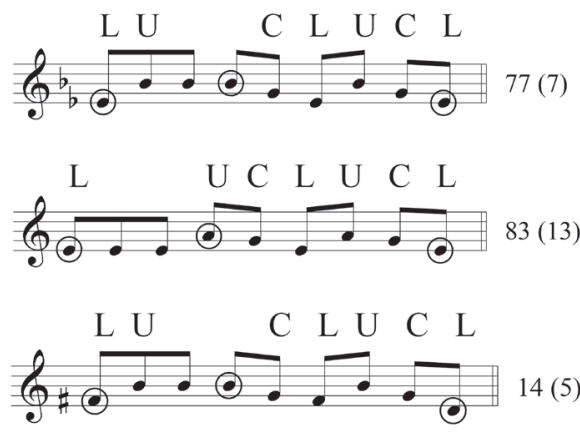

Figure 8. The interchangeability of the scale notes: a) $B b=A b$; b) $E=E b, A=A b$; c) $E b=E=F$ \#, $A=B b=B, D=E b=E$. 
A comparison of the variants based on the same melodic contours also allows us to trace one more aspect of the scale development process - the different ways of increasing the number of notes in the scale. Presuming the 3-note scales to be of older origin than the 4- and 5-note scales, we should take into account two theoretical possibilities as to how the new scale notes could be added to the scales: (1) the extension of the scale in an upward and/or downward direction by adding neighbouring notes; and (2) the filling of the 'gaps' in the scale (by 'gaps' I mean the intervals bigger than the major second). The analysis shows that in the case of the Seto shepherd tune we are dealing mainly with the second manner of development. Figure 9 compares four variants of the first line of the tune. The first variant is based on the 3-note scale, the others on the 4- and 5-note scales. Comparison of the variants reveals that the new scale notes (designated by circles) emerge mainly as 'passing tones' between the scale notes $\mathrm{G}$ and $\mathrm{B} / \mathrm{Bb}$ and between $\mathrm{E} / \mathrm{E} b$ and $\mathrm{G}$. In the final example, there is also the 'neighbouring tone' $\mathrm{C}$.
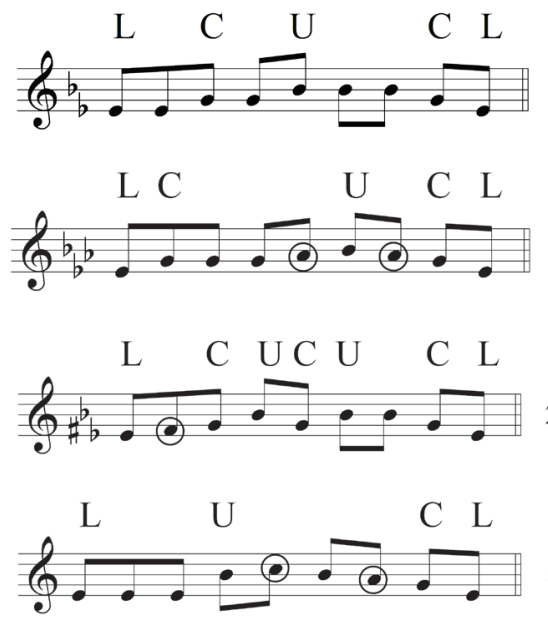

Figure 9. The 'filling' of the 'gaps'.

According to the author's listening experience, the pitch of the 'passing' and 'neighbouring tones' can be especially unstable, making their notation quite problematic. In this article, however, I proceed from the published transcriptions and from the analytical decisions made by the transcribers. The control of these transcriptions, which could also include their acoustical measurement, is a subject for future research. 


\section{DISCUSSION AND CONCLUSIONS}

It is interesting now to explore how the results of the analysis carried out in this study correspond to existing hypotheses in the literature on scales and melody in early traditional music.

To begin with the German classification from the era of comparative musicology, which divided the types of archaic melody into Engmelodik, Pendelmelodik, Treppenmelodik, and Fanfarenmelodik, one can find features of three of these in the Seto shepherd songs. The tune variants in the range of the perfect fourth (e.g. with the scale E-G-A) can be defined as a narrow-range melody (Engmelodik). The tune variants based on the scale that coincides with the major triad (Eb$\mathrm{G}-\mathrm{B} b$ ) may at first glance seem to belong to the fanfare type (Fanfarenmelodik). At the same time, however, the melodic motion where the melody leaps the interval of a third up and down from the central tone and also the prevalence of the ascending-descending melodic contour may recall the movement of the pendulum (Pendelmelodik).

The possibility of finding such different melodic types in the performances of the same song points not only to the great variability of the tune in question, but also to the insufficient reliability of the exterior parameters of the melodic contour as a ground for judgements about the principles of melodic thinking. It is very difficult to reveal these principles on the basis of the individual performances, and thus the researcher should take into account the great number of tune variants and try to understand the inner logic that links them. In the Seto shepherd songs the logical principle behind the melody formation would appear to be the contradistinction of the lower and upper supporting tones with the pivotal central tone (the principal tone of the mode). Such a functional pattern would seem to be the stable feature of the Seto shepherd tune, whereas the archaic way of musical thinking manifests itself in the fact that the intervals between the three main tones of the scale may vary to a considerable extent and that the filling of these intervals by passing tones is optional.

Bruno Nettl, in his book "Music in Primitive Culture" (1956), also suggested his own classification of the types of melodic contour in early traditional music. It seems that in proposing three main types of melodic motion - 'ascending', 'descending', and 'undulating movement' (Nettl 1956: 51) - he proceeded not so much from the specific features of archaic melody, but rather from the logical generalization of the potentially possible melodic situations. ${ }^{10}$ Choosing which of these three types corresponds better to the Seto shepherd tune, I would point to the third one - the 'undulating movement' - or even its particular form called the 'pendulum-type melodic movement'. The variants of the tune analysed conform to Nettl's assertion that "both undulating and pendulum-type melodies usually 
conclude with a descending progression, as does most primitive music" (Nettl 1956: 52). The ascending-descending melodic contour (LCUCL), which is the most widespread in the Seto shepherd songs, is also mentioned by Nettl as one of the 'specialized types': "A melodic contour that resembles an arc is another specialized type. The melody rises and falls in roughly equal amounts, the curve ascending gradually to a climax and then dropping off" (Nettl 1956: 53).

Another interesting question regarding early traditional music is the interrelation of melodic contour and scale structure. According to the statistics concerning the use of the different scales and melodic contours in the Seto shepherd songs, among the melodic contours one model definitely predominates, whereas among the scale structures there are no strong preferences. This finding is in line with the statement that "frequently the Gestalt of a melody contour is more important than the precise intervals of a melody" (Ambrazevičius \& Budrys \& Višnevska 2015: 27). A comparison of the melodic variants shows that the same contour can be realized with different scales and melodic intervals. However, the analysis also reveals that the melodic contours may vary within the same tune type, whereas the main functional model of the scale (mode) structure - the three pitch level structure - is constant. Together with the characteristic ascending-descending contour, many other particular contours occur, including the most elementary descending contour (see the last musical example in Figure 7). Though the scheme in Figure 7 demonstrates that every contour can be derived from the descending one, this hardly allows us to assume that the simple descending contour was historically the oldest.

A further attempt to classify the types of early traditional melody is undertaken by Eduard Alekseyev in his book Early Folklore Intonation: The Pitch Aspect (1986). ${ }^{11}$ This classification seems to be the most convincing of the existing schemes because the author proceeds from the inner logic of the melody formation, describing not so much the particular melodic contours as the principles of their generation. Alekseyev separates three types of early traditional melody building, which he designates by the letters of the Greek alphabet $\alpha-, \beta-$, and $\gamma$-melody (Alekseyev 1986: 53). The first two types are especially archaic: the $\alpha$-melody is based on the contrasting opposition of both the pitch registers and voice registers, where the timbral difference is more important than the intervallic coordination; the $\beta$-melody is characterized as the 'primary (initial) glissando' (pervichnoe glissandirovanie) (ibid.: 64), where the pitch is "constantly changing - mostly sliding down" (ibid.: 206). The third principle, the $\gamma$-melody, according to Alekseyev's description, "better ... corresponds with our ideas of definite pitch-levels as the tone-material of singing; yet, in contrast with melody-making in terms of crystallized tonality, the $\gamma$-degrees are subject to considerable, though gradual, changes in respect of pitch when a melodic 
unit is repeated, so that different versions of a tone may be integrated into one transformative degree [emphasis added]" (ibid.).

It seems that the $\gamma$-principle best characterizes the pitch organization of the Seto shepherd songs because their intonation is mostly quite definite, although the pitch of the scale notes can vary at different moments in a performance (the degree of the pitch stability of the scale notes depends largely on the individual performer). The unusual diversity of the scale structures in the different performances of the shepherd tune is evidence that its intervallic relations are not yet settled. In this tune, we can speak about the existence of a certain number of scale notes as categories of modal thinking, but the pitch zones of their realization are very wide. This last phenomenon causes some specific problems in the notation of these songs, since the transcriber has to notate the same scale degree using different notes.

At the same time, in the most archaic variants of the Seto shepherd songs (i.e. the performances based on the 3-note scales) we can also find traces of the more ancient $\alpha$-melody and some small manifestations of the $\beta$-melody. According to Alekseyev, these three principles are not mutually exclusive and unrelated; on the contrary, they can be integrated and form different combinations (Alekseyev 1986: 206). In the tune in question, the $\alpha$-principle manifests itself in the juxtaposition of three pitch levels (the main variable scale notes) mostly with the interval of a third between them. The filling of the thirds with the sliding intermediate tones ${ }^{12}$ seems to be a manifestation of the $\beta$-principle. Although the third is not a very wide melodic interval, it is wide enough as a step between neighbouring scale notes (especially if there are no seconds in the scale) to create a bigger pitch contrast in the melodic motion than in the case of the, so to speak, 'filled' scales. Developing Alekseyev's ideas further, we may consider the existence of two processes in the gradual comprehension of the pitch dimension in early traditional music: (1) by means of the pitch and timbral contrast of more or less distant tones (the $\alpha$-principle), which led to the gradual establishment of the basic tones of the modal system; and (2) by means of the vocal glissando (possibly between the basic tones) (the $\beta$-principle), which led to the formation of stepwise motion (where a step is the interval of a second). Both processes can lead to the formation of the so-called $\gamma$-melody, where the more definite pitch-levels (scale notes) as the musical tone-material exist. In the Seto shepherd songs, we can observe both these processes at work.

As noted above, the analysis of the melodic contours helps us better to understand the processes of scale formation; however, the result of these processes, i.e. the intervallic structure of the scales, is also of a great interest to researchers. Although studies show that in early traditional vocal music the intervallic structure of the scale varies much more than the melodic contour, 
the scale structure does not appear to be chaotic and some consistent patterns emerge. Many ideas have been put forward in this field of research, some of which should be mentioned in connection with the Seto shepherd tune. In discussing these, we must take into account the limitations of research based on the analysis of the published musical transcriptions, which can include both accidental and systematic misrepresentations of the pitch relations. In this sense we are merely raising some questions here, more reliable answers to which may be found in the course of future acoustical research.

One of the widespread theories with regard to scale structure in early traditional music is the assumption as to the special role of the perfect consonances as a universal of musical thinking (see, e.g., Nettl 1956: 54). At first glance, the scales of the Seto shepherd songs might seem to confirm this assumption: the majority of the scales have the range of a perfect fifth, while there are also scales with the range of a perfect fourth, and finally there are scales which coincide with the major triad. Nevertheless, in my opinion, neither perfect consonances nor the major triad as acoustical phenomena have a constitutive function in the formation of the shepherd songs' scales. This conclusion is based on the fact that in this tune the principal tone of the mode is located in the middle of the scale, and the intervals that are likely to have any importance for the performers are those between the central tone and the upper or lower supporting tones. The lower and upper scale notes are not directly related; they rather surround the central tone. Thus we can conclude that the most characteristic, supporting intervals in this tune are the thirds, the size of which fluctuates between the minor and major third.

There remains the question of the nature of the 'major triad' in this tune: is this a true 'fanfare' or a 'false' one? On the one hand, the scale Eb-G-Bb occurs in seven performances recorded by six different singers (see the data in Figure 2 ), which is sufficient to recognize that this structure is not accidental (for comparison, the minor triad never occurs as a 3-note scale, while the diminished triad is found in only two performances by the same singer). To these seven performances can be added 17 melodic variants based on the partly or entirely 'filled' scales in the range of the perfect fifth, with the scale notes Eb, G, and Bb as the main tones of the mode. On the other hand, there are 14 performances with the 3-, 4-, and 5-note scales in the range of the diminished fifth and six performances based on the anhemitonic trichord E-G-A, which suggests that there is no strong preference for the 'major triad'. The cases where the scale structure varies during the same performance (see the analytical part of this article) confirm this conclusion.

Why then does the 'major triad' in its 'filled' or 'unfilled' form occur so frequently? It seems that the explanation could be connected with the one-three- 
semitone mode, which is characteristic of the older layer of the Seto multipart songs. This scale, which can be transcribed as D-Eb-F\#-G-A\#-B or D-Eb-F\#-G$\mathrm{B} b-\mathrm{C} b$, comprises the notes of the major triad - Eb, G and $\mathrm{B} b$. The 4-note fragment of this scale Eb-F\#-G-Bb is found in three performances of the shepherd song, which supports the surmise about the relationship between shepherd songs and multipart songs. It would be logical to assume that the structures $\mathrm{E} b-\mathrm{F} \sharp-\mathrm{G}-\mathrm{B} b$ and $\mathrm{E} b-\mathrm{G}-\mathrm{B} b$ might be connected with each other. If this assumption is true, it is a further reason to believe that the 'major triad' in the shepherd tune is merely a coincidence. This opinion is supported by the fact that the harmonic sonority $\mathrm{E} b-\mathrm{G}-\mathrm{B} b$ (A\#) is very rare in the Seto multipart songs, and, in any case, it is not a structural sonority (the structural role in this mode is fulfilled by the 'augmented triads' Eb-G-B and D-F\#-A\# or, more commonly, the major thirds from these two functional complexes). The perfect fifths and fourths are structurally insignificant in the songs based on the one-three-semitone scale, although we can find such intervals between the scale notes. This mode is built from the trichords in the range of the major third (e.g. F\#-G-A\# and Eb-F\#-G), and the major third is its most important interval.

All these observations lead us to the conclusion that the 'major triad' in the Seto shepherd songs is in substance a 'false fanfare' and that the principle of consonance did not play a significant role in the formation of the Seto shepherd tune and its scales. However, in the more recent times, the musical thinking of singers might differ from that of their predecessors, and they could give new meanings to the ancient musical structures.

Another interesting question that has been raised in the literature on the scales in traditional music concerns the use of equal or unequal intervals between adjacent scale notes. The most widely accepted theory states that the intervallic structure of the scale should be asymmetrical. But there is also another viewpoint with regard to this question. According to recent research on the scales found in Lithuanian traditional music, "the universal of unequal scale steps, however, seems to be an overestimation, since equidistant scales ... are also widely known in different musical cultures all over the world" (Ambrazevičius \& Budrys \& Višnevska 2015: 22). The acoustical analysis carried out in their book led the authors to the assumption that equidistant (equitonic) scales might be much more widespread in traditional music than might appear in published music transcriptions since the conflict between emic and etic perceptions of the scales can lead to erroneous interpretations (ibid.: 29-31, 169-170). The authors also suppose that the equitonic principle is more ancient than the asymmetrical one (e.g. the well-known diatonic scales): "Most probably, one of the most important and perhaps one of the historically earliest principles is roughly equidistant pitch arrangement" (ibid.: 23; see also 418). This last hypothesis is especially important in the context of the present research. 
In the Seto shepherd songs, the question about asymmetrical and equidistant scale structures should be examined under two aspects: on the one hand, in relation to the intervals between the three main scale notes (which may be the only scale notes in the simplest tune variants) and, on the other, in relation to the 'filling' (passing) tones between the three main scale notes. Nettl, in the above-mentioned book on 'primitive music', writes that in the 3-note scales unequal intervals are usually used: "The two intervals are seldom identical; usually a major second is combined with a minor second or a minor third. In the very few known cases where two thirds are used, they are heterogeneous" (Nettl 1956: 48). Thus, Nettl advocates here the opinion as to the preference for an asymmetrical structure in the primary scales. The statistics for the intervallic structure of the 3-note scales in the Seto shepherd songs confirm Nettl's observation: among such scales the combinations of a major third and minor third (Eb-G-Bb) and minor third and major second (E-G-A) prevail, although there are also two cases where the thirds are of equal size (E-G-Bb). However, among the 4- and 5-note scales, the structures with minor thirds between the three main scale notes are not infrequent, which means that, as the frame of the mode, the equidistant structure is quite acceptable.

In relation to the 'filling' notes between the central tone and the upper and lower supporting tones some preferences can be found, but we cannot be sure to whom these preferences belong - whether it is the performers or the transcribers. According to the statistics in Figure 2, the minor third G-Bb is most often filled by the note A (18 performances); however, the structure $\mathrm{G}-\mathrm{A} b-\mathrm{B} b$ also occurs in 11 performances. The lower minor third E-G is more frequently filled by the note $\mathrm{F}$ (15 performances); the structure E-F\#-G occurs only in three cases. The major thirds, both upper and lower, are always filled equitonically by major seconds (except in three performances based on the one-three-semitone scale $\mathrm{E} b-\mathrm{F} \#-\mathrm{G}-\mathrm{B} b)$. From these statistics, we may note that the seconds above and below the central tone are mostly major. However, the findings of the above-mentioned research by Ambrazevičius, Budrys, and Višnevska make me cautious in drawing conclusions with regard to this topic. Firstly, when using the Western music notation, it is quite difficult to divide the minor third equitonically, and the difference in pitch in comparison with the well-tempered tuning system would be too small to be noticed by the transcribers. Secondly, according to my aural impressions, the passing tones in the Seto shepherd songs are often sung with a sliding intonation, meaning that the transcribers had to make quite difficult decisions when writing down these notes. Clearly, the only way to clarify this issue would be an acoustical analysis of the sound recordings.

A further question with regard to the intervallic structure of the Seto shepherd songs is the location of the wider and narrower intervals in the scale. In 
the analytical part of this article, a pattern was detected, according to which the wider intervals are situated lower in the scale than the narrower intervals. This concerns mainly the location and size of the thirds. If there are two different thirds in the scale, the major third is situated under the central tone and the minor third above it. If there is only one third, it is always located in the lower part of the scale. If the thirds are filled by passing tones, the upper third is filled first. This situation may be a latent manifestation of the so-called 'proportional scales', which occur in some archaic traditional music cultures (Alekseyev 1976: 91; Ambrazevičius \& Budrys \& Višnevska 2015: 13). Some manifestations of 'proportional scales' are also found in the Seto multipart songs (Ambrazevičius \& Pärtlas 2011: 14), but this topic requires more detailed investigation.

Taking into account the unusually great diversity of the intervallic structures of the Seto shepherd songs' scales, the question arises as to the extent to which the theoretical differentiation between all these structures is justified. It is obvious that many of these differences may be caused merely by the looseness of intonation and the wide 'realization zones' of the scale notes, both of which are characteristic of many archaic traditional music styles. In this connection, Nettl asserted that the accuracy of the intonation depends on the size of the intervals between the adjacent scale notes: the bigger the intervals, the less precise the intonation (Nettl 1956: 50). Nevertheless, it seems that each performer of the Seto shepherd songs has a more or less definite model of the scale structure, and the singers are quite consistent in the realization of their models. These models may be local or even individual, and the degree of variability in the realization of the model depends on the particular performers and their cultural background, with greater variability obviously characterizing more archaic musical thinking. It is reasonable to assume that these various scale models are not entirely accidental and are therefore worth investigating from the point of view of the processes of scale formation. The individual variability of the tuning could be a topic for future research.

In conclusion, I would propose one further hypothesis as to the possible manner in which the scale development in the Seto shepherd songs took place - one which may have a wider application in the field of early traditional vocal music. To this purpose, I would use the scheme of the perceptual levels of musical scales by Dowling and Harwood (1986) (Figure 10). ${ }^{13}$ I borrowed this scheme from the above-cited research by Ambrazevičius, Budrys, and Višnevska (2015: 9), where it is employed as the theoretical basis of the discussion about the scales in traditional music. The difference in my approach is that I try to apply this scheme to the historical process of scale formation.

The scheme describes four levels of perception of the musical scales, beginning with a pitch continuum, which is the first stage of the pitch hierarchy 


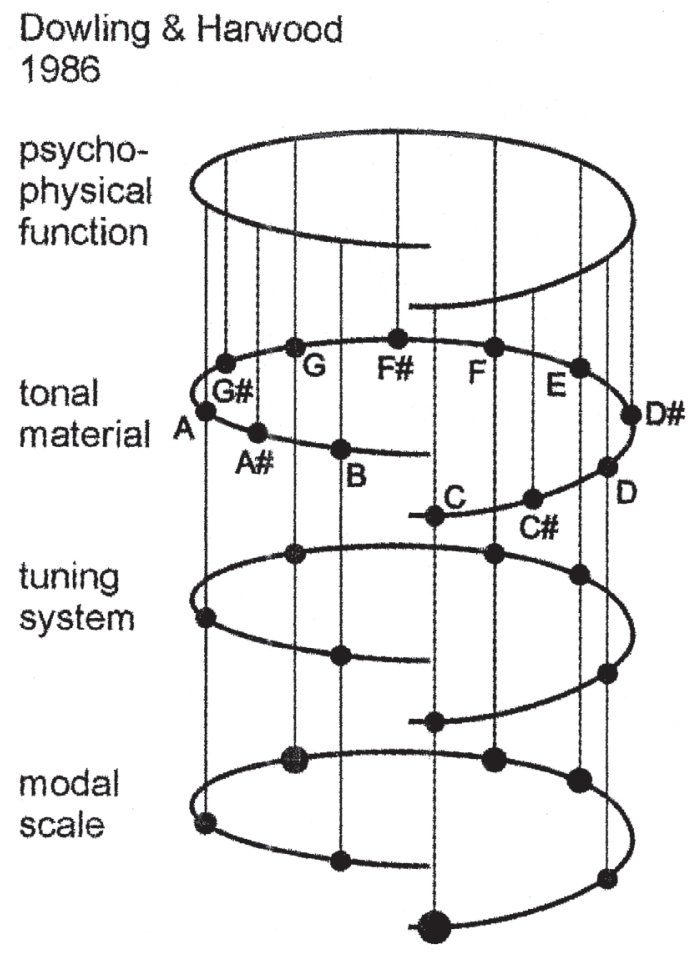

Figure 10. The perceptual levels of musical scales (based on Dowling and Harwood 1986).

based on the psychophysical functions, and ending with the developed hierarchic system of the mode where each scale note has a certain modal function (in the scheme, this level is named a 'modal scale'). The intermediate levels are the establishment of the minimum distance between possible pitch categories (a 'tonal material') and the formation of the intervallic structure of the scale (a 'tuning system').

Although from a cognitive viewpoint the perceptual levels of the musical scales presented in such an order make a logically perfect scheme, we can assume that historically the development of musical thinking hardly proceeded in this way. Taking these levels as developmental stages we could, on the contrary, move along this scheme in an ascending direction; the psychophysical level, however, as the natural basis for pitch perception is fundamental and not part of the developmental process. This assumption is based on the premise that in the process of its development the functional structure of the mode does not need a well-established system of pitch relations. 
In the light of the analysis carried out in this paper, it seems that the initial stage of the scale formation might have been the establishment of the small number of the main modal functions, which were embodied in the higher or lower pitch zones without fixing their exact intervallic relation (the level of the 'modal scale' in Figure 10). In the Seto shepherd songs, as we noted above, there are three main functions - the central principal tone and the upper and lower supporting tones. Initially the timbral and pitch contrast of such mutable scale degrees was of greater importance than the exact pitch relations. However, we can imagine that subsequently the intervals between the main scale notes were recognized by the singers more exactly until more or less stable intervallic models were established (the level of the 'tuning system'). After that - or maybe at the same time - there were attempts to fill the intervals between the main scale notes by passing tones. The filling occurred in different ways, and this process can be considered as an intuitive search for the possible pitch categories (the level of the 'tonal material'). The appearance of the less important passing tones made the functional structure of the mode more complex. The intervallic fixation of the additional scale notes and their functioning as units of pitch measurement might have led to the establishment of more stable intervals between the main scale notes.

The hypothetical developmental model presented here is not meant to be the only historical explanation of scale formation; it does, however, fit well with the musical material analysed in this paper and could hopefully have wider application. The three stages described are unlikely to have occurred successively; rather, they probably overlapped each other. Furthermore, in the Seto shepherd songs, this process was never completed, which is why we can speak about archaic musical thinking in this case. However, the various performances of the Seto shepherd songs gathered in Anu Vissel's collection may demonstrate different stages of the scale formation process.

This 'polyphony' of historical layers is characteristic of traditional musical cultures in general; it is an essential feature of traditional musical thinking, and it can appear even in a single artefact. A close analysis of the musical texts, especially when a great number of variants are available, allows us to uncover this layering and sometimes to 'excavate' evidence about the very ancient structures of musical thinking that are present in them.

\section{ACKNOWLEDGEMENTS}

This research was supported by the Centre of Excellence in Estonian Studies (CEES, European Regional Development Fund) and is related to research project IUT 12-1 (Estonian Research Council). 


\section{NOTES}

1 Under 'contemporary ethnomusicology' I here mean the predominant mainstream English-language ethnomusicological tradition.

2 Among the rare exceptions is the recent thorough monograph on musical scales in Lithuanian traditional music: Ambrazevičius \& Budrys \& Višnevska 2015. The theoretical questions of the musical modality were also developed in the works by Timo Leisiö (2002a, b).

3 Such modal systems may probably be defined as 'additive tonal systems' (see Jouste \& Niemi 2002: 254-257).

4 One of the reasons why a semitone is preferable as a standard unit of intervals' measurement is its connection with the much criticized but still the only readable system of musical notation broadly used in ethnomusicology.

5 For instance, it is noticed that the scale notes that significantly disagree with the 12 TET system are often perceived by the transcribers as more unstable than others (Ambrazevičius 2005).

6 The sound-recording of this performance is available on the CD Helmine: Mikitamäe leelokoor (1999).

7 I here speak about the scales as they were notated by the transcribers. This circumstance lowers to some extent the value of the exact figures, but I still believe that the general conclusions made as a result of the analysis are also valid for actual song performances.

8 Bruno Nettl, in his book Music in Primitive Culture, pointed to the need for the analysis of the melodic movement to gain a better understanding of the scale formation: "Melodic movement is a particularly significant factor in scale analysis, one which has been somewhat neglected in favor of the study of scales" (Nettl 1956: 51).

9 All examples of the melodic contours (the first lines of the melostrophes) in this and the following figures originate from Vissel's collection. The numbers beside the examples refer to the numbers of the transcriptions in this publication; the numbers in the brackets show the numbers of the melostrophes. In the short musical examples and schemes, the melody is presented without the text for two reasons: firstly, in the Seto shepherd songs the verbal text generally influences neither the structure of the musical scales nor the melodic contour; secondly, the schemes are easier to follow if they are not overcharged with redundant information.

${ }^{10}$ Such an approach explains the fact that, as Nettl himself noticed, the first of these three types of melodic contour actually occurs very seldom in a 'primitive' melody.

11 This is a literal translation of the book's title (Rannefol'klornoe intonirovanie). In the English summary of the book, the free rendering is preferred - "The Pitch Nature of Primitive Singing" (Alekseyev 1986: 203).

12 This observation is founded on my aural impressions, and it should be checked by the aid of an acoustical analysis. 
${ }^{13}$ It is important to emphasize that I use this scheme here mainly as a 'source of inspiration', being aware of the differences between the original conception and my developments: firstly, the scheme by Dowling and Harwood was designed for the diatonic scale of the Western tonal system, whereas I apply it to the scales of traditional music; secondly, this scheme originally depicts the final result of scale development, whereas I interpret the levels of the scheme as the stages in the process of development of the modal system.

\section{REFERENCES}

Alekseyev 1976 = Alekseev, Eduard. Problemy formirovaniia lada (na materiale iakutskoi narodnoi pesni). [Issues Related to the Formation of Musical Mode (on the Basis of Yakut Traditional Songs).] Moscow: Muzyka. Available at http://olonkho.info/ eduard_alekseyev/pfl/index.html, last accessed on February 2, 2017.

Alekseyev 1986 = Alekseev, Eduard. Rannefol'klornoe intonirovanie: Zvukovysotnyi aspekt. [Early Folklore Intonation: The Pitch Aspect.] Moscow: Sovetskii kompozitor. Available at http://olonkho.info/eduard_alekseyev/rfi/index.html, last accessed on February 2, 2017.

Ambrazevičius, Rytis 2005. The Perception and Transcription of the Scale Reconsidered: Several Lithuanian Cases. The World of Music, Vol. 47, No. 2, pp. 31-53. DOI: $10.2307 / 41699644$.

Ambrazevičius, Rytis \& Budrys, Robertas \& Višnevska, Irena 2015. Scales in Lithuanian Traditional Music: Acoustics, Cognition, and Contexts. Kaunas: Kaunas University of Technology.

Ambrazevičius, Rytis \& Pärtlas, Žanna 2011. Searching for the "Natural" Origins of the Symmetrical Scales: Traditional Multipart Setu Songs. Journal of Interdisciplinary Music Studies, Vol. 5, No. 1, pp. 1-17. Available at http://www.musicstudies.org/ spring2011.html, last accessed on February 2, 2017.

Dowling, W. Jay \& Harwood Dane L. 1986. Music Cognition. Orlando, FL: Academic Press.

Jouste, Marko \& Niemi, Jarkko 2002. Monochronic and Additive Principles in North Eurasian Song Styles. In: Irina Nurieva (ed.) Etnomuzykovedenie Povolzh’ia i Urala $v$ areal'nykh issledovaniiakh. [Ethnomusicology of Volga and Ural Regions in Regional Research.] Izhevsk: Udmurtskii institut istorii, iazyka i literatury UrO RAN, pp. 250-282.

Leisiö, Timo 2002a. Folk Music Styles and Prehistory in Western Eurasia: A Modal Approach to Analysis. In: Kyösti Julku (ed.) The Roots of Peoples and Languages of Northern Eurasia IV. Oulu: Societas Historiæ Fenno-Ugricæ, pp. 151-175.

Leisiö 2002b = Leisie, Timo. Terminologicheskie i logicheskie problemy muzykovedeniia. [Terminological and Logical Problems of Music Studies.] In: Irina Nurieva (ed.) Etnomuzykovedenie Povolzh'ia i Urala v areal'nykh issledovaniiakh. [Ethnomusicology of Volga and Ural Regions in Regional Research.] Izhevsk: Udmurtskii institut istorii, iazyka i literatury UrO RAN, pp. 283-297. 
Nettl, Bruno 1956. Music in Primitive Culture. Cambridge: Harvard University Press. Available at https://archive.org/details/MusicInPrimitiveCulture, last accessed on February 2, 2017.

Pärtlas, Žanna 1997. Tähelepanekuid setu laulu laadiehitusest ja mitmehäälsusest. [Some Remarks about Modal Structure and Polyphony in the Setu Songs.] Teater. Muusika. Kino, No. 1, pp. 23-28. Available at http://www.digar.ee/arhiiv/et/ perioodika/41109, last accessed on February 2, 2017.

Pärtlas, Žanna 2006a. "Sümmeetrilised laadid” ja monointervalliline mitmehäälsus vokaalses rahvamuusikas: mõningaid paralleele setu ja Lõuna-Venemaa rahvalaulu vahel. ["Symmetrical Modes" and Mono-Interval Polyphony in Vocal Folk Music: Some Parallels between the Setu and Southern Russian Folk Song.] In: Aado Lintrop (ed.) Regilaul - esitus ja tõlgendus. [Regilaul - Performance and Interpretation.] Tartu: Eesti Kirjandusmuuseum, pp. 219-248.

Pärtlas, Žanna 2006b. Foundations of the Typology of Setu Folk Tunes. In: Triinu Ojamaa \& Andreas Kalkun (eds.) Individual and Collective in Traditional Culture. Individuaalne ja kollektiivne traditsionaalses kultuuris. Tartu: Eesti Kirjandusmuuseum, pp. 38-46.

Pärtlas, Žanna 2010a. Setu Multipart Singing: Comparison of Written Sources and Sound Recordings. In: Susanne Ziegler \& Urban Bareis (eds.) Historical Sources and Source Criticism. Stockholm: Svenskt Visarkiv, pp. 227-237.

Pärtlas 2010b = Piartlas, Zhanna. O probleme vospriiatiia i notatsii ladov s podvizhnymi stupeniami: eksperiment A. M. Listopadova i setuskie arkhaicheskie zvukoriady. [About the Perception of Modes with Mutable Tones: Experiment by A. M. Listopadov and the Ancient Setu Scales.] In: N. Al'meeva (comp.) \& A. Nekrylova \& A. Romodin (eds.) Fol'klor i my: traditsionnaia kul'tura v zerkale ee vospriiatii. Sb. nauch. statei, posviashchennyi 70-letiiu I. I. Zemtsovskogo. Part 1. [Folklore and We: Traditional Culture in the Mirror of Its Perceptions.] St Petersburg: Izd-vo RIII, pp. 158-170.

Sarv 1980 = Sarv, Iaan. Rasshifrovka setuskogo mnogogolosiia pri pomoshchi mnogokanal'noi studiinoi apparatury. [About the Notation of Setu Folk Songs by Means of Multi-Track Studio-Apparatus.] In: I. Riuitel (comp.) Finno-ugorskii muzykal'nyi fol'klor i vzaimosviazi s sosednimi kul'turami. [Finno-Ugric Musical Folklore and the Interrelations with the Neighbouring Cultures.] Tallinn: Eesti Raamat, pp. 103-126.

Sarv, Vaike (ed.) 1999. Helmine: Mikitamäe leelokoor. [Leelo Choir from Mikitamäe.] Tartu: Eesti Kirjandusmuuseum \& Eesti Keele Instituut. CD.

Vissel, Anu 1982. Eesti karjaselaulud I: Setu karjaselaulud. [Estonian Shepherd Songs I: Setu Shepherd Songs.] Tallinn: Keele ja Kirjanduse Instituut.

Žanna Pärtlas is Senior Researcher at the Estonian Academy of Music and Theatre, Tallinn, Estonia. She studied musicology at the Rimsky-Korsakov State Conservatory, St. Petersburg, where she received her $\mathrm{PhD}$ in 1992.

zhanna@ema.edu.ee 